\title{
Late starting treadmill exercise improves spatial leaning ability through suppressing CREP/BDNF/TrkB signaling pathway following traumatic brain injury in rats
}

\author{
II-Gyu Ko ${ }^{1,2}$, Sung-Eun Kim, ${ }^{1,2}$ Lakkyong Hwang ${ }^{1,2}$, Jun-Jang Jin ${ }^{1,2}$, Chang-Ju Kim ${ }^{1,2}$, Bo-Kyun Kim³, Hong Kim ${ }^{4, *}$ \\ 1Department of Physiology, College of Medicine, Kyung Hee University, Seoul, Korea \\ ${ }^{2}$ Kohwang Medical Research Institute, Kyung Hee University, Seoul, Korea \\ ${ }^{3}$ Department of Emergency Technology, College of Health Science, Gachon University, Incheon, Korea \\ ${ }^{4}$ Department of Oriental Sports Medicine, College of Biomedical Science, Daegu Haany University, Gyeongsan, Korea
}

Traumatic brain injury (TBI) causes deficit in spatial learning and memory function. Physical activity ameliorates neurological dysfunction after TBI. We investigated the effect of late starting treadmill exercise on spatial learning ability in relation with CAMP-response element binding protein (CREB)/brain-derived neurotrophic factor (BDNF) signaling pathway using TBI rats. For this study, radial 8-arm maze test, TUNEL (terminal deoxynucleotidyl transferase-mediated dUTP nick end labeling) staining, caspase-3 immunohistochemistry, and western blot for Bax, Bcl-2, BDNF, tyrosine kinase B (TrkB), CREB, and phosphorylated CREP ( $p$-CREB) were performed. TBI was induced by an electromagnetic-controlled cortical impact. The rats in the exercise groups were scheduled to run on a treadmill for 30 min once a day for 8 weeks starting 3 weeks after TBI. TBI impaired spatial learning ability and increased caspase- 3 expression in the hippocampal dentate gyrus. TBI enhanced Bax expression and suppressed $\mathrm{Bcl}-2$ expression in the hippocampus. TBI increased BDNF and TrkB expressions, resulted in the enhancement of $p$-CREB/CREB ratio in the hippocampus. However, treadmill exercise improved spatial learning ability, decreased caspase- 3 expression, suppressed Bax expression, and increased Bcl2 expression. Treadmill exercise alleviated TBI-induced over-expression of BDNF and TrkB, which suppressed phosphorylation of CREB in the hippocampus. In the present study, late starting treadmill exercise improved spatial learning ability through suppressing TBI-induced activation of CREB/BDNF/TrkB signaling pathway after TBI.

Keywords: Traumatic brain injury, Treadmill exercise, Spatial learning ability, Brain-derived neurotrophic factor, cAMP-response element binding protein, Apoptosis

\section{INTRODUCTION}

Traumatic brain injury (TBI) is known to results in deficits in spatial learning and memory function (Kim et al., 2010a; Shen et al., 2013). In generally, TBI causes cell death and neurological dysfunction through direct physical disruptions of tissue or pathways (primary injury) as well as through delayed biochemical changes induced by trauma (secondary injury). TBI triggers a complex cascade of apoptotic events which can contribute to delayed secondary injury processes.

Apoptosis, or programmed cell death, is a physiological form of cell death that is important for normal embryologic development and cell turnover in adult organisms. Apoptosis plays a crucial role in delayed neuronal loss after both acute and chronic brain injury. In the TBI-induced apoptosis, Bcl-2 family plays as the key executioner of apoptosis and caspase family acts as the final pathway of apoptosis (Kim et al., 2010a; Park et al., 2014; Zhang et al., 2005).

Brain-derived neurotrophic factor (BDNF) and its receptor, tyrosine kinase $\mathrm{B}(\mathrm{TrkB})$, are broadly expressed in the mammalian brain. BDNF/TrkB signaling is critical for neuronal survival, morphogenesis, and plasticity (Numakawa et al., 2010). Activa-
${ }^{*}$ Corresponding author: Hong Kim (i) https://orcid.org/0000-0001-8740-3600 Department of Oriental Sports Medicine, College of Biomedical Science, Daegu Haany University, 1 Haanydae-ro, Gyeongsan 38610, Korea Tel: +82-53-819-1468, Fax: +82-53-819-1264, E-mail: joshua@dhu.ac.kr Received: April 16, 2018 / Accepted: May 15, 2018
This is an Open Access article distributed under the terms of the Creative Commons Attribution Non-Commercial License (http://creativecommons.org/licenses/by-nc/4.0/) which permits unrestricted non-commercial use, distribution, and reproduction in any medium, provided the original work is properly cited. 
tion of BDNF/TrkB signaling protects against TBI through cAMP-response element binding protein (CREB) cascade (Wu et al., 2014). CREB is a transcription factor in the brain and it is implicated in neuronal survival and spatial learning ability (Griesbach et al., 2009; Shen et al., 2013).

Physical activity ameliorates neurological dysfunction after TBI through inhibition of apoptosis, attenuation of neuro-inflammation and oxidative stress, and improving neuro-restoration (Archer et al., 2012; Piao et al., 2013). However, the effect of exercise on TBI is controversial, depending on the time of the start of the exercise.

Farmer et al. (2004) defined that appropriate time window for voluntary exercise after brain injury is dependent on injury severity in which an increase in injury severity prolongs the period of nonresponsiveness to voluntary exercise. Voluntary exercise endogenously upregulates BDNF and enhances recovery when it was delayed after TBI, however, when exercise was rapidly administered to TBI, the molecular response to exercise is disrupted and recovery might be delayed (Griesbach et al., 2004). Early started exercise may be deleterious by exacerbating posttraumatic symptomatology and disrupting restorative processes (Griesbach, 2011).

In the present study, we investigated the effects of late starting treadmill exercise on spatial learning ability in relations with CREB- BDNF signaling pathway using TBI rats. For this study, radial 8-arm maze test, TUNEL (terminal deoxynucleotidyl transferase-mediated dUTP nick end labeling) staining, caspase- 3 immunohistochemistry, and western blot for $\mathrm{Bax}, \mathrm{Bcl}-2$, BDNF, TrkB, CREB, and p-CREB were performed.

\section{MATERIALS AND METHODS}

\section{Animals and treatments}

Male Sprague-Dawley rats, weighing $90 \pm 5 \mathrm{~g}$ (4 weeks old), were used for the experiments. The experimental procedures were performed in accordance with the animal care guidelines of the National Institutes of Health and the Korean Academy of Medical Sciences. This study was approved by the Kyung Hee University Institutional Animal Care and Use Committee (Seoul, Korea, KHUASP[SE]-17-089). The animals were randomly divided into the following four groups ( $\mathrm{n}=10$ in each group): sham-operation group, sham-operation and treadmill exercise group, TBI-induced group, and TBI-induced and treadmill exercise group.

\section{Induction of TB|}

TBI was made based on the previous study (Park et al., 2014). For the induction of TBI, the rats were anesthetized with Zoletil
$50(10 \mathrm{mg} / \mathrm{kg}$, intraperitoneally; Vibac Laboratories, Carros, France). A circular craniotomy $(5.0 \mathrm{~mm})$ was performed using a Dremel motor tool and a specially designed drill bit that prevented damage to the meninges and cortex $(2.4 \mathrm{~mm}$ lateral to the midline, and $4.2 \mathrm{~mm}$ posterior to the coronal suture). The contusion injury was created with an electromagnetic contusion device (Impact One, Stereotaxic Impactor; MyNeurolab, St. Louis, MO, USA) using a sterile stainless steel impactor tip (3.0-mm diameter) that was activated at a velocity of $3.00 \mathrm{~m} / \mathrm{sec}$. The impactor tip was positioned above the cortex, and resulted in a $2.5-\mathrm{mm}$ compression to the cortex.

\section{Exercise protocol}

The rats in the exercise groups were scheduled to run on a treadmill for 30 min once a day for 8 weeks (56 days) starting 3 weeks (21 days) after TBI induction. The rats in the exercise groups were forced to run on a motorized treadmill (Columbus Instruments, Columbus, OH, USA). The exercise load consisted of running at speeds of $2 \mathrm{~m} / \mathrm{min}$ for the first $5 \mathrm{~min}, 5 \mathrm{~m} / \mathrm{min}$ for the next $5 \mathrm{~min}$, and then $8 \mathrm{~m} / \mathrm{min}$ for the last $20 \mathrm{~min}$, at an inclination of $0^{\circ}$.

\section{Radial 8-arm maze test}

Spatial learning ability was determined using a radial 8-arm maze apparatus, according to the previously described method (Ji et al., 2015; Kim et al., 2010b). The rats were deprived of water for $24 \mathrm{hr}$ and allowed to explore the water for $5 \mathrm{~min}$. The test was conducted on the 76 days after TBI-induced operation ( 55 days after starting the treadmill exercise). The time spent in seeking water at the end of the arms was counted. The test was terminated when a rat found water in all 8 arms or when over 8 min elapsed. Re-entry into the previously visited arms was counted as an error. The number of correct choice before the first error was also counted.

\section{Tissue preparation}

The animals were sacrificed after last treadmill running. The rats were anesthetized using Zoletil $50(10 \mathrm{mg} / \mathrm{kg}$, intraperitoneally; Vibac Laboratories), transcardially perfused with $50 \mathrm{mM}$ phosphate-buffered saline, and fixed with a freshly prepared solution consisting of $4 \%$ paraformaldehyde in $100 \mathrm{mM}$ phosphate buffer ( $\mathrm{pH}, 7.4)$. Brains were dissected, and storage overnight same fixative, then it was transferred to $30 \%$ sucrose for cryoprotection. For the immunohistochemistry, the slices were coronal sectioned at $40 \mu \mathrm{m}$ thick using a cryostat (Leica, Nussloch, Germany). 


\section{Immunohistochemistry for caspase-3}

Immunohistochemistry was conducted to evaluate the caspase-3 expression in the hippocampal dentate gyrus, according to the previously described method (Kim et al., 2014; Ko et al., 2009). Free-floating tissue sections were incubated overnight with mouse anti-caspase-3 antibody (1:500; Santa Cruz Biotechnology, Santa Cruz, CA, USA) at a dilution of 1:1,000, and the sections were then incubated for $1 \mathrm{hr}$ with biotinylated anti-mouse antibody for caspase-3 (1:200; Vector Laboratories, Burlingame, CA, USA). Next, the sections were incubated with avidin-biotin-peroxidase complex (Vector Laboratories) for $1 \mathrm{hr}$ at room temperature. The sections were incubated in a reaction mixture consisting of $0.03 \%$ DAB (3,3'-diaminobenzidine) and $0.03 \%$ hydrogen peroxide for $5 \mathrm{~min}$. The sections were finally mounted onto gelatin-coated slides. The slides were air dried overnight at room temperature, and coverslips were mounted using Permount (Fisher Scientific, New Jersey, NJ, USA).

\section{Western blotting}

Western blot for the expressions of Bax, Bcl-2, BDNF, TrkB, CREB, and p-CREB were performed, according to the previously described method (Kim et al., 2014; Ko et al., 2009). The hippocampal tissues were homogenized on ice, and lysed in a lysis buffer containing $50 \mathrm{mM}$ HEPES (pH, 7.5), $150 \mathrm{mM} \mathrm{NaCl}, 10 \%$ glycerol, $1 \%$ Triton X-100, $1 \mathrm{mM}$ phenylmethylsulfonyl fluoride, $1 \mathrm{mM}$ EGTA, $1.5 \mathrm{mM} \mathrm{MgCl} 2 \cdot 6 \mathrm{H}_{2} \mathrm{O}, 1 \mathrm{mM}$ sodium orthovanadate, and $100 \mathrm{mM}$ sodium fluoride. Protein content was measured using a Bio-Rad colorimetric protein assay kit (Hercules, CA, USA). Protein samples ( $30 \mu \mathrm{g})$ were separated on sodium dodecyl sulfate-polyacrylamide gel and transferred onto a nitrocellulose membrane. The membranes were incubated with $5 \%$ skim milk in Tris-buffered saline containing 0.1\% Tween-20 and then incubated overnight at $4^{\circ} \mathrm{C}$ with the following primary antibod- ies: mouse anti- $\beta$-actin antibody, mouse anti-Bcl-2 antibody, mouse anti-Bax antibody, rabbit anti-BDNF antibody, rabbit anti-TrkB antibody, rabbit anti-CREB antibody, rabbit anti-p-CREB antibody (1:1,000; Santa Cruz Biotechnology). Subsequently, membranes were incubated for $1 \mathrm{hr}$ with attempt secondary antibodies (1:2,000; Vector Laboratories), and ban detection was performed using the enhanced chemiluminescence detection kit (Santa Cruz Biotechnology).

\section{Data analysis}

The detected bands of Bax, Bcl-2, BDNF, TrkB, CREP, and p-CREP were calculated densitometrically using Molecular Analyst ver. 1.4.1 (Bio-Rad). The area of the granular layer of the hippocampal dentate gyrus from each slice was measured by Image-Pro Plus image analysis system (Media Cyberbetics Inc., Silver Spring, MD, USA). The number of caspase-3-positive cells in the hippocampal dentate gyrus was counted hemilaterally under a light microscope (Olympus, Tokyo, Japan), and expressed as the number of cells per square millimeter of granular area in the hippocampal dentate gyrus.

Differences among the groups were evaluated using IBM SPSS Statistics ver. 21.0 (IBM Co., Armonk, NY, USA). For the comparison among the groups, one-way analysis of variance was performed followed by Duncan post hoc test. All values are expressed as the mean \pm standard error of the mean. Statistically significant differences were established at $P<0.05$.

\section{RESULTS}

\section{Effect of treadmill exercise on the spatial learning ability in the radial 8-arm maze test}

The time of complete performance, the number of correct, and the number of error in the radial 8-arm maze test are presented in
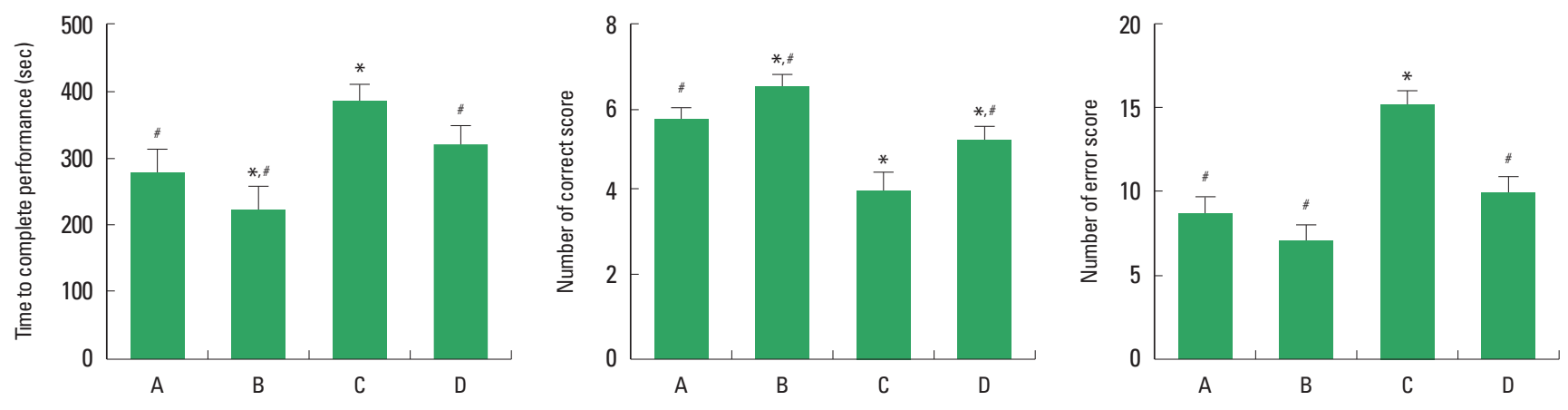

Fig. 1. Effect of treadmill exercise on complete time (upper panel), correct number (middle panel), and error number (lower panel) in the radial 8-arm maze test. A, Sham-operation group; B, sham-operation and treadmill exercise group; $C$, traumatic brain injury (TBI)-induced group; D, TBI-induced and treadmill exercise group. ${ }^{*} P<0.05$ compared to the sham-operation group. ${ }^{\#} P<0.05$ compared to the TBI-induced group. 
Fig. 1. These results showed that induction of TBI increased completed time, decreased number of correct, and increased number of error. In contrast, treadmill exercise for 8 weeks decreased completed time, increased correct number, and reduced error number in the TBI-induced rats.

\section{Effect of treadmill exercise on caspase-3 expression in the hippocampal dentate gyrus}

Photomicrographs of caspase-3-positive cells in the hippocampal dentate gyrus are presented in Fig. 2. These results showed that induction of TBI enhanced caspase-3 expression in the hippocampal dentate gyrus. In contrast, treadmill exercise for 8
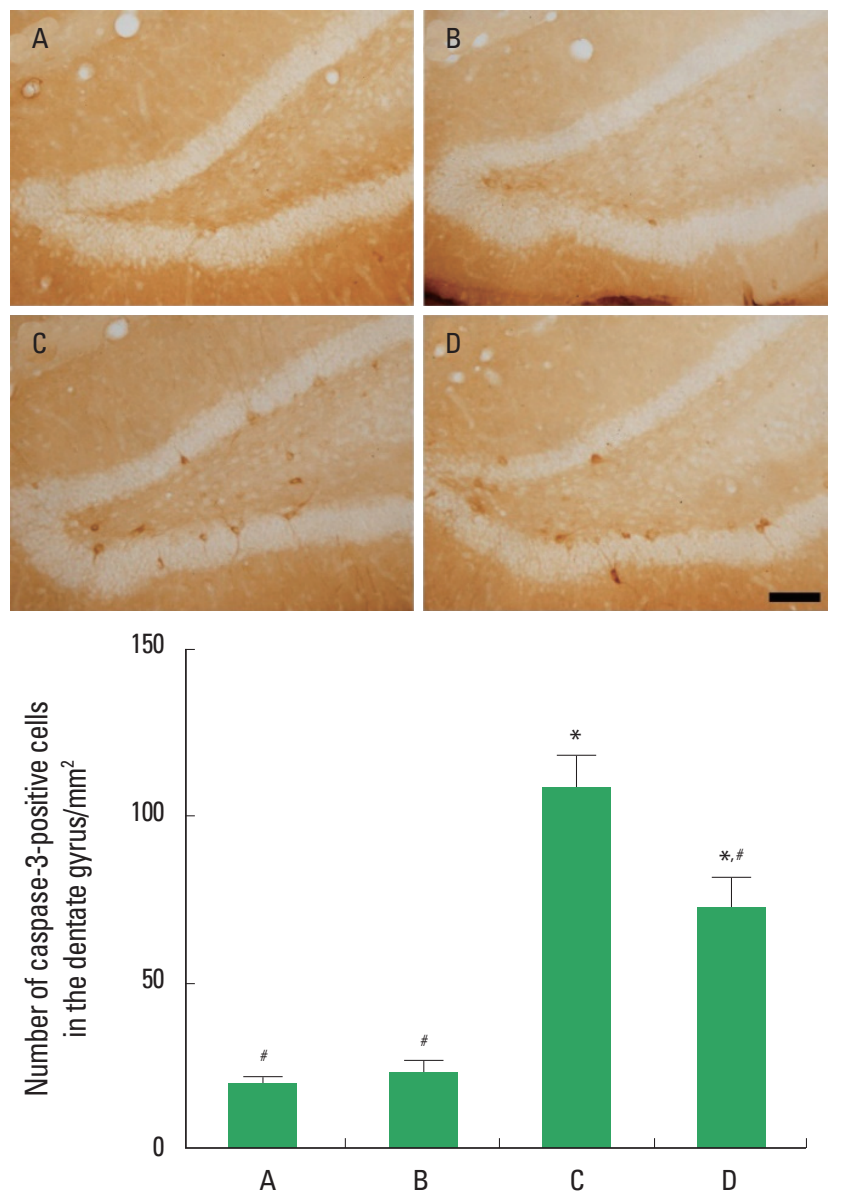

Fig. 2. Effect of treadmill exercise on caspase-3 expression in the hippocampal dentate gyrus. Upper panel: Photomicrographs of caspase-3-positive cells in the hippocampal dentate gyrus. The scale bar represents $150 \mu \mathrm{m}$. Lower panel: Number of caspase-3-positive cells in each group. A, Sham-operation group; $B$, sham-operation and treadmill exercise group; $C$, traumatic brain injury (TBI)-induced group; $\mathrm{D}$, TBl-induced and treadmill exercise group. ${ }^{*} P<0.05$ compared to the sham-operation group. ${ }^{\sharp} P<0.05$ compared to the TBI-induced group. weeks suppressed caspase-3 expression in the TBI-induced rats.

\section{Effect of treadmill exercise on Bax and Bcl-2 expressions in the hippocampus}

The relative expressions of $\mathrm{Bax}$ and $\mathrm{Bcl}-2$ in the hippocampus are presented in Fig. 3. These results showed that induction of TBI enhanced the expression Bax and enfeebled the expression of $\mathrm{Bcl}-2$. Therefore, induction of TBI enhanced the ratio of Bax to $\mathrm{Bcl}-2$, represents facilitation of apoptosis in the hippocampus. However, treadmill exercise during 8 weeks suppressed the expression of $\mathrm{Bax}$ and enhanced the expression of $\mathrm{Bcl}-2$, resulting in suppression of $\mathrm{Bax}$ to $\mathrm{Bcl}-2$ ratio in the TBI-induced rats.

\section{Effects of treadmill exercise on BDNF and TrkB expressions in the hippocampus}

The relative expressions of BDNF and TrkB in the hippocampus are presented in Fig. 4. These results showed that induction of TBI enhanced BDNF and TrkB expressions in the hippocampus. In contrast, treadmill exercise during 8 weeks suppressed BDNF and TrkB expressions in the TBI-induced rats.

\section{Effects of treadmill exercise on the ratio of $p$-CREB to CREB in the hippocampus}

The relative expressions of CREB and $\mathrm{p}-\mathrm{CREB}$ are presented in Fig. 5. These results showed that induction of TBI enhanced the ratio of $\mathrm{p}-\mathrm{CREB}$ to $\mathrm{CREB}$ in the hippocampus. In contrast, treadmill exercise during 8 weeks suppressed the ratio of $\mathrm{p}-\mathrm{CREB}$ to CREB in the TBI-induced rats.

\section{DISCUSSION}

There are many reports that exercise counteracts the cognitive deficits associated with the brain injury (Griesbach et al., 2009; Kim et al., 2010a; Ko et al., 2009). In the present study, longer complete time, less correct choice, and more error choice were observed in the TBI-induced rats, representing that TBI deteriorated spatial learning ability. Treadmill exercise shortened complete time, increased correct number, and decreased error number in the TBI-induced rats, showing that treadmill exercise alleviated TBI-induced impairment of spatial learning ability. Such a memory improving effect of physical exercise has been reported in many other studies. Griesbach et al. (2004) reported that physical exercise activated neuroplasticity mechanisms within the hippocampus and counteracted cognitive deficit induced by experimental TBI. Treadmill exercise alleviated TBI-induced short-term 


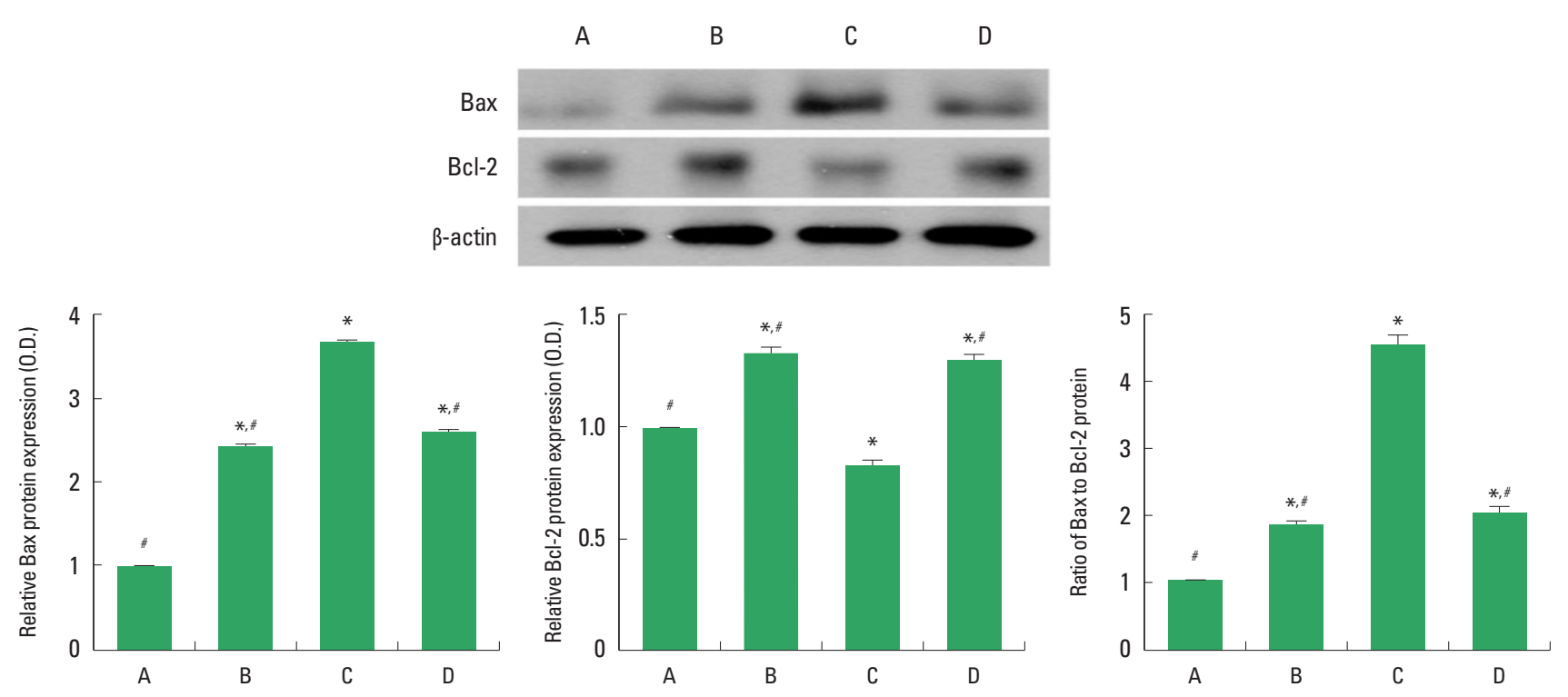

Fig. 3. Effects of treadmill exercise on Bax and Bcl-2 expressions in the hippocampus. Upper panel: The results of band detection using the enhanced chemiluminescence detection kit. Actin was used as an internal control (46 kDa). Lower panel: The relative expressions of Bax and Bcl-2 in each group. A, Sham-operation group; B, sham-operation and treadmill exercise group; $\mathrm{C}$, traumatic brain injury (TBI)-induced group; D, TBI-induced and treadmill exercise group. ${ }^{*} P<0.05$ compared to the sham-operation group. ${ }^{*} P<0.05$ compared to the TBI-induced group.

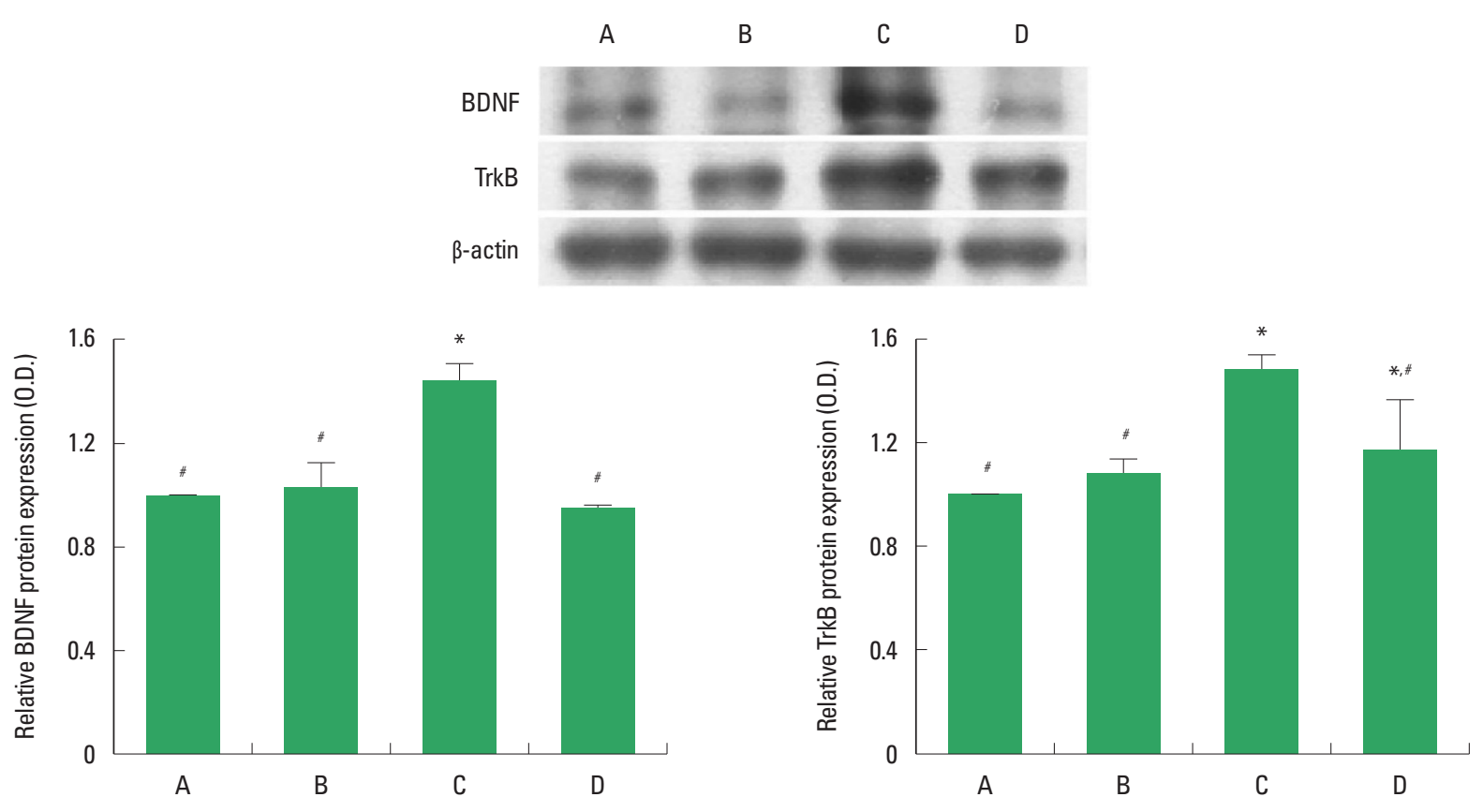

Fig. 4. Effects of treadmill exercise on brain-derived neurotrophic factor (BDNF) and tyrosine kinase B (TrkB) expressions in the hippocampus. Upper panel: The results of band detection using the enhanced chemiluminescence detection kit. Actin was used as an internal control (46 kDa). Lower panel: The relative expressions of BDNF and TrkB in each group. A, Sham-operation group; B, sham-operation and treadmill exercise group; C, traumatic brain injury (TBI)-induced group; D, TBI-induced and treadmill exercise group. ${ }^{*} P<0.05$ compared to the sham-operation group. ${ }^{\#} P<0.05$ compared to the TBI-induced group.

memory impairment by suppressing TBI-induced apoptotic neuronal cell death (Kim et al., 2010a; Park et al., 2014).

During the process of secondary injury of TBI, apoptosis-regu- latory factors are implicated in the susceptibility of neurons to cell death (Zhao et al., 2015). Bax and Bcl-2 are two distinct members in the regulation of apoptosis. $\mathrm{Bcl}-2$ is functionally characterized 

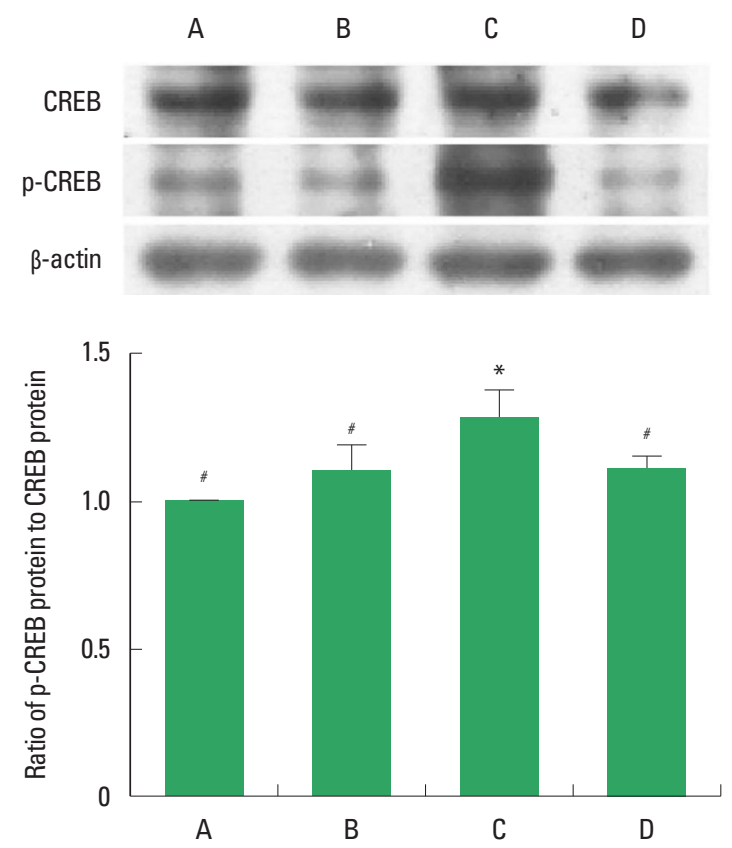

Fig. 5. Effects of treadmill exercise on cAMP-response element binding protein (CREB) and phosphorylated CREP (p-CREB) expressions in the hippocampus. Upper panel: The results of band detection using the enhanced chemiluminescence detection kit. Actin was used as an internal control ( $46 \mathrm{kDa}$ ). Lower panel: The relative ratio of $p$-CREB to CREB in each group. A, Sham-operation group; $B$, sham-operation and treadmill exercise group; $C$, traumatic brain injury (TBI)-induced group; D, TBI-induced and treadmill exercise group. ${ }^{*} P<0.05$ compared to the sham-operation group. ${ }^{*} P<0.05$ compared to the TBI-induced group.

as the apoptosis-suppressing factor, whereas, Bax is considered as the apoptosis-promoting factor. The ratio of $\mathrm{Bax}$ to $\mathrm{Bcl}-2$ is a crucial factor determining whether cells undergo apoptosis, and brain insults disrupt the ratio of $\mathrm{Bax}$ to $\mathrm{Bcl}-2$ (Strauss et al., 2004). Many studies have shown that treadmill exercise prevented apoptotic cell death in the hippocampus and facilitated recover from brain injury (Kim et al., 2010a; Park et al., 2014; Zhao et al., 2015). In the present study, the number of caspase-3-positive cells in the hippocampal dentate gyrus were increased following TBI. In addition, Bax expression was up-regulated and Bcl-2 expression was down-regulated following TBI, resulting in increment of Bax to Bcl-2 ratio in the hippocampus. The present results showed that secondary injury of TBI increased apoptosis in the hippocampus. However, treadmill exercise suppressed the TBI-induced caspase- 3 expression in the hippocampal dentate gyrus. Treadmill exercise remarkably suppressed Bax expression and increased $\mathrm{Bcl}-2$ expression, resulting in decrement of $\mathrm{Bax}$ to $\mathrm{Bcl}-2$ ratio in the hippocampus. The present results showed that treadmill exercise suppressed TBI-induced apoptosis in the hippocam- pus. Our present results support the previous reports that treadmill exercise enhanced short-term and spatial memory through suppressing TBI-induced apoptotic neuronal cell death in the hippocampus (Kim et al., 2010a; Zhao et al., 2015). Increment of $\mathrm{Bcl}-2$ by physical exercise inhibits the release of cytochrome $\mathrm{c}$ from the mitochondria to the cytosol, resulting in suppression of neuronal apoptosis (Hiebert et al., 2015; Itoh et al., 2011).

BDNF is altered by TBI, and this increment of BDNF might be considered as one of the mechanisms promoting neuroprotection and neuronal repair after damage (Guan et al., 2003). Similarly, up-regulation of cell proliferation and/or neurogenesis during pathological situations has been suggested as a compensatory response to TBI-induced apoptotic cell death (Kernie and Parent, 2010).

In the present study, the expressions of BDNF and TrkB in the hippocampus were increased at 11 weeks after TBI induction. The expressions of BDNF and TrkB in neurons are linked with the phosphorylation of CREB (Griesbach et al., 2009; Wu et al., 2014). BDNF/TrkB signaling is critical for promoting neuronal survival following brain damage. Activation of BDNF/TrkB signaling protects against TBI via the phosphatidylinositide 3-kinase (PI3K)/Akt, but not extracellular regulated kinase pathway, and this protective effect may be amplified via the PI3K/Akt/CREB cascade (Wu et al., 2014). Such being the cases, the present study showed that long-term after TBI induction, the ratio of $\mathrm{p}-\mathrm{CREB} /$ CREB in the hippocampus was enhanced. In the present results, phosphorylation of CREB was facilitated through activation of $\mathrm{BDNF} / \mathrm{TrkB}$ pathway. These mechanisms contribute for acceleration of neuronal repair following TBI. In the previous studies, neurotrophic factors was up-regulated in traumatic insult at few hours after the brain lesion and this up-regulation lasted for 4 weeks (Feliciano et al., 2014; Madathil et al., 2010).

It is still unclear whether excessive expression of neurotrophic factors including BDNF promotes or delays functional recovery from TBI. Physical exercise suppressed overexpression of neurotrophic factors following nerve injury (Byun et al., 2005; Griesbach, 2011). In the present study, treadmill exercise ameliorated TBI-induced overexpression of BDNF and TrkB, which suppressed phosphorylation of CREB in the hippocampus. Our study maintained recovery period for 3 weeks after TBI, and treadmill exercise lasted during 8 weeks, it was progressed total 11 weeks after induction of TBI. There is still controversy on the starting time of exercise after brain injury. If brain is energetically compromised, exercise-induced increment in metabolic demand may negatively impact neurological recovery (Griesbach et al., 2012). 
Initiation of physical exercise in the first few days after TBI caused suboptimal induction of neuroplasticity/neuroprotective pathways such as BDNF/TrkB pathway, and consequently resulted in poor functional or neuropathological outcomes (Zhao et al., 2015).

Here in this study, we demonstrated that treadmill exercise for 11 weeks starting 3 weeks after TBI also improved spatial learning ability by suppressing apoptotic neuronal cell death in the hippocampus. These effects of treadmill exercise appeared through suppressing TBI-induced activation of CREB/BDNF/TrkB signaling pathway. Based on the present results, late starting treadmill exercise is also a potential useful therapeutic strategy for the treatment of TBI.

\section{CONFLICT OF INTEREST}

No potential conflict of interest relevant to this article was reported.

\section{ACKNOWLEDGMENTS}

This research was supported by the Basic Science Research Program through the National Research Foundation of Korea (NRF) funded by the Ministry of Education, Science and Technology (NRF-2011-0021908).

\section{REFERENCES}

Archer T, Svensson K, Alricsson M. Physical exercise ameliorates deficits induced by traumatic brain injury. Acta Neurol Scand 2012;125:293302.

Byun YH, Lee MH, Kim SS, Kim H, Chang HK, Lee TH, Jang MH, Shin MC, Shin MS, Kim CJ. Treadmill running promotes functional recovery and decreases brain-derived neurotrophic factor mRNA expression following sciatic crushed nerve injury in rats. J Sports Med Phys Fitness 2005;45:222-228.

Farmer J, Zhao X, van Praag H, Wodtke K, Gage FH, Christie BR. Effects of voluntary exercise on synaptic plasticity and gene expression in the dentate gyrus of adult male Sprague-Dawley rats in vivo. Neuroscience 2004;124:71-79.

Feliciano DP, Sahbaie P, Shi X, Klukinov M, Clark JD, Yeomans DC. Nociceptive sensitization and BDNF up-regulation in a rat model of traumatic brain injury. Neurosci Lett 2014;583:55-59.

Griesbach GS. Exercise after traumatic brain injury: is it a double-edged sword? PM R 2011;3(6 Suppl 1):S64-72.

Griesbach GS, Hovda DA, Gomez-Pinilla F. Exercise-induced improve- ment in cognitive performance after traumatic brain injury in rats is dependent on BDNF activation. Brain Res 2009;1288:105-115.

Griesbach GS, Hovda DA, Molteni R, Wu A, Gomez-Pinilla F. Voluntary exercise following traumatic brain injury: brain-derived neurotrophic factor upregulation and recovery of function. Neuroscience 2004;125: 129-139.

Griesbach GS, Tio DL, Vincelli J, McArthur DL, Taylor AN. Differential effects of voluntary and forced exercise on stress responses after traumatic brain injury. J Neurotrauma 2012;29:1426-1433.

Guan J, Bennet L, Gluckman PD, Gunn AJ. Insulin-like growth factor-1 and post-ischemic brain injury. Prog Neurobiol 2003;70:443-462.

Hiebert JB, Shen Q, Thimmesch AR, Pierce JD. Traumatic brain injury and mitochondrial dysfunction. Am J Med Sci 2015;350:132-138.

Itoh T, Imano M, Nishida S, Tsubaki M, Hashimoto S, Ito A, Satou T. Exercise inhibits neuronal apoptosis and improves cerebral function following rat traumatic brain injury. J Neural Transm (Vienna) 2011;118: 1263-1272.

Ji ES, Kim YM, Shin MS, Kim CJ, Lee KS, Kim K, Ha J, Chung YR. Treadmill exercise enhances spatial learning ability through suppressing hippocampal apoptosis in Huntington's disease rats. J Exerc Rehabil 2015;11:133-139.

Kernie SG, Parent JM. Forebrain neurogenesis after focal Ischemic and traumatic brain injury. Neurobiol Dis 2010;37:267-274.

Kim DH, Ko IG, Kim BK, Kim TW, Kim SE, Shin MS, Kim CJ, Kim H, Kim KM, Baek SS. Treadmill exercise inhibits traumatic brain injury-induced hippocampal apoptosis. Physiol Behav 2010a;101:660-665.

Kim SE, Ko IG, Kim BK, Shin MS, Cho S, Kim CJ, Kim SH, Baek SS, Lee EK, Jee YS. Treadmill exercise prevents aging-induced failure of memory through an increase in neurogenesis and suppression of apoptosis in rat hippocampus. Exp Gerontol 2010b;45:357-365.

Kim YH, Sung YH, Lee HH, Ko IG, Kim SE, Shin MS, Kim BK. Postnatal treadmill exercise alleviates short-term memory impairment by enhancing cell proliferation and suppressing apoptosis in the hippocampus of rat pups born to diabetic rats. J Exerc Rehabil 2014;10:209-217.

Ko IG, Shin MS, Kim BK, Kim SE, Sung YH, Kim TS, Shin MC, Cho HJ, Kim SC, Kim SH, Kim KH, Shin DH, Kim CJ. Tadalafil improves shortterm memory by suppressing ischemia-induced apoptosis of hippocampal neuronal cells in gerbils. Pharmacol Biochem Behav 2009;91: 629-635.

Madathil SK, Evans HN, Saatman KE. Temporal and regional changes in IGF-1/IGF-1R signaling in the mouse brain after traumatic brain injury. J Neurotrauma 2010;27:95-107.

Numakawa T, Suzuki S, Kumamaru E, Adachi N, Richards M, Kunugi H. BDNF function and intracellular signaling in neurons. Histol Histopathol 2010;25:237-258. 
Park MS, Oh HA, Ko IG, Kim SE, Kim SH, Kim CJ, Kim HB, Kim H. Influence of mild traumatic brain injury during pediatric stage on shortterm memory and hippocampal apoptosis in adult rats. J Exerc Rehabil 2014;10:148-154.

Piao CS, Stoica BA, Wu J, Sabirzhanov B, Zhao Z, Cabatbat R, Loane DJ, Faden AI. Late exercise reduces neuroinflammation and cognitive dysfunction after traumatic brain injury. Neurobiol Dis 2013;54:252263.

Shen X, Li A, Zhang Y, Dong X, Shan T, Wu Y, Jia J, Hu Y. The effect of different intensities of treadmill exercise on cognitive function deficit following a severe controlled cortical impact in rats. Int J Mol Sci 2013; 14:21598-21612.

Strauss KI, Narayan RK, Raghupathi R. Common patterns of bcl-2 family gene expression in two traumatic brain injury models. Neurotox Res 2004;6:333-342.

Wu CH, Hung TH, Chen CC, Ke CH, Lee CY, Wang PY, Chen SF. Post-injury treatment with 7,8-dihydroxyflavone, a TrkB receptor agonist, protects against experimental traumatic brain injury via PI3K/Akt signaling. PLoS One 2014;9:e113397.

Zhang X, Chen Y, Jenkins LW, Kochanek PM, Clark RS. Bench-to-bedside review: apoptosis/programmed cell death triggered by traumatic brain injury. Crit Care 2005;9:66-75.

Zhao Z, Sabirzhanov B, Wu J, Faden AI, Stoica BA. et al. Voluntary exercise preconditioning activates multiple antiapoptotic mechanisms and improves neurological recovery after experimental traumatic brain injury. J Neurotrauma 2015;32:1347-1360. 